\title{
Editorial to the first issue
}

\author{
Meng-Hiot Lim • Steven Gustafson • \\ Natalio Krasnogor • Yew-Soon Ong
}

Published online: 29 January 2009

(C) Springer-Verlag 2009

The last 2 decades have seen the emergence of a large number of computational intelligence techniques derived from the natural sciences. New nature-inspired problem-solving paradigms emerged that are based on Darwinian evolution, entomology, condensed matter physics, neurobiology, immunology, etc. These paradigms, in turn, popularized search methodologies such as Genetic Algorithms, Genetic Programming, Evolution Strategies, Particle Swarm Optimization, Ant Colony Optimization, Simulated Annealing, Neural Networks, Artificial Immune Systems, etc. At the same time, other search methodologies such as Tabu-search, Scatter Search, GRASP, etc, remained "metaphor-less". A war-of-the-method ensued and continues, often it is the case that each of these search paradigms has a niche flagship publication where the latest advances within the paradigm are presented. The IEEE Transactions on Evolutionary Algorithms or its twin publication on Neural Networks, the Evolutionary Computation journal, the journal of Genetic Programming and Evolvable Machines, the Swarm Intelligence Journal, etc, are examples of scientific outlets for work derived from nature-inspired principles, while the Journal of Heuristics, the Journal of Soft Computing-A Fusion of Foundations, Methodologies and Applications or the more recent International Journal of Metaheuristics are examples of publications where the research emphasis is not necessarily on natural computation.

M.-H. Lim ( $₫)$ · Y.-S. Ong

Nanyang Technological University, Singapore, Singapore

e-mail: emhlim@ntu.edu.sg

S. Gustafson

GE Global Research, Niskayuna, New York, USA

N. Krasnogor

University of Nottingham, Nottingham, UK
In recent years, a new trend has emerged across a variety of metaheuristic studies, whether nature-inspired or metaphorless, that recognizes that true innovation and breakthrough in optimization and machine learning often arises from the amalgamation of, sometimes, disparate concepts borrowed from different optimisation and machine learning paradigms. These "hybrid" algorithms are often times called Memetic Algorithms. "Memetic" Algorithms as a terminology, thus serves to unify studies arising from disparate optimization and machine learning traditions. The need for a new journal on Memetic Computing is thus clear: until now no single journal identified the importance and central role of hybridization for search methodologies. Hybrid algorithms have traditionally been seen from the perspective of the "host" paradigm, e.g., evolutionary algorithms, Ant Colony optimization, Artificial Immune System. Indeed, the goal of this journal is to bring center stage the multi-faceted roles of hybridization thus breaking with the main orthodoxy that lies at the heart of many other, albeit excellent, publications. Moreover, the Memetic Computing journal recognizes the critical needs for a more fluid, direct and sustained dialogue between the metaheuristics and nature-inspired search methodologies with the approximate and exact algorithms research communities. A situation not dissimilar to the one mentioned above occurs between heuristic methodologies and exact and/or approximate methods, namely, often the most successful exact algorithms based approaches to real world problems are the result of sophisticated composition of heuristics (either nature-inspired or metaphor-less) with exact or approximate mathematical programming techniques. Yet, a scientific venue specially dedicated to the systematic study of the interplay between metaheuristics and exact/ approximation methods did not, until now, exist. The creation of a new journal such as ours should serve not only an existing need for organizing a research field along clear and 
well established lines (as we suggest above), but also brings forth new possibilities.

Universal Darwinism theory as coined by Richard Dawkins provides a unifying framework governing the evolution of any complex systems. In particular, "Universal Darwinism" suggests that evolution is not exclusive to biological systems, i.e., it is not confined to the narrow context of the genes, but applicable to any complex systems that exhibit the principles of inheritance, variation and selection, fulfilling the traits of an evolving system. For example, the emerging science of memetics attempts to explain cultural, cognitive and psychosocial phenomena with biological evolutionary analogues. The term "meme" was also introduced and defined by Dawkins in 1989 as "the basic unit of cultural transmission, or imitation", and in the English Oxford Dictionary as "an element of culture that may be considered to be passed on by non-genetic means". We believe that the idea of going beyond the mimicry of biological evolution towards a multi-track evolution of software and hardware artifacts transcends, indeed, the field of combinatorial and continuous optimization.

The aim of this journal is to capture the growing research trends in Memetic Computing. We are optimistic that the Memetic Computing journal will be a useful channel for dissemination of work that captures the richness and diversity of an emerging frontier in computational techniques. The overreaching goals of this new journal are thus:

1. To be an outlet for high quality research in hybrid metaheuristics for optimization, control and design in continuous and discrete optimization domains regardless of whether these are nature-inspired or metaphor-less methodologies. We seek to dissolve the barriers separating metaheuristics, exact and approximation algorithms research and to bring forth a renewed impetus towards the investigation and understanding of promising new hybrid algorithmic technologies.

2. To go beyond current search methodologies towards innovative research on the emergence of cultural artifacts such as game, trade and negotiation strategies and, more generally, rules of behavior as they apply to, for example, robotic, multi-agent and artificial life systems.

3. Ultimately, Memetic Computing aspires to serve as a focal publication where the latest results in Natural Computation, Artificial Intelligence, Machine Learning, Operational Research, exact and approximate optimization and Natural Sciences (e.g. cognitive, animal and insect's behavior, etc.) combined together in novel ways in order to transcend the intrinsic limitations of a single discipline.

In this inaugural issue of Memetic Computing journal, we are proud to include five papers which are illustrative of the range of techniques we hope to feature in this journal. The first paper by Yu et al. addresses the important issue of diversity in evolutionary algorithms. They give an extensive coverage of both direct and indirect immigrants schemes, substantiated with experiments to understand the behaviors of the two schemes before proposing a modified approach that draws on the strengths and merits of the two. We believe that the results of the paper are significant considering that dynamic optimization problems will be an important area of future research. In the second article of this issue, Gutin and Parapetyan attempt to formalize a theoretical framework that is useful for the design and analysis of optimization heuristics. Their approach, based on the notion of signed graphs, may serve as a starting point for addressing one of the core issues in optimization heuristics, essentially a theoretical framework to explain why some heuristics work and some do not. The paper by Santana, Larranaga and Lozano gives a comprehensive treatment of issues pertaining to estimation of distribution algorithms. They survey key open research topics on discrete estimation of distribution algorithms that we hope will foster further research. The fourth paper by Bacardit, Burke and Krasnogor serves as a timely reminder of the potential behind genetics based machine learning approaches. In their paper, the authors demonstrated how large datasets, arising in this instance from bioinformatics and systems biology problems, can effectively be dealt with by generating rules that are more effective in abstracting the necessary information embedded in the dataset resulting in high accuracy classifications with rich explanatory power. The final paper in this issue by Hasan et al. shows that memetic algorithms can be effective in tackling classical optimization problems in operations research, in particular job-shop scheduling. Their work reinforces the notion that incorporation of memes in the form of local searches can produce results that are competitive to other existing algorithms reported in literature.

We are grateful to the authors who contributed their best work for this inaugural issue of Memetic Computing. Moreover, we would like to thank the editors who managed the review process in a professional manner as well as the reviewers for their invaluable contributions by carefully reviewing the papers as to ensure their publication worthiness. The authors no doubt benefitted from their critical comments and feedback. We look forward to receiving more research articles with theoretical and applications oriented themes that serve to advance the field of memetic computing. Our journal will also welcome articles in the form of short technical correspondences and book reviews. We would welcome suggestions and ideas on how the journal could be improved as to effectively cater for the needs of researchers and practitioners working at the interface of the various communities this journal seeks to serve. 